Andrés Moreno Roca, Luciana Armijos Acurio, Ruth Jimbo Sotomayor*, Carlos Céspedes Rivadeneira, Carlos Rosero Reyes and Carlos López Ayala

\title{
Cohort study of the overall survival of patients with pancreatic cancer in a hospital of specialties of Quito-Ecuador in the period 2007-2017
}

https://doi.org/10.1515/iss-2020-0030

Received October 11, 2020; accepted January 11, 2021; published online May 3, 2021

\section{Abstract}

Objectives: Pancreatic cancers in most patients in Ecuador are diagnosed at an advanced stage of the disease, which is associated with lower survival. To determine the characteristics and global survival of pancreatic cancer patients in a social security hospital in Ecuador between 2007 and 2017.

Methods: A retrospective cohort study and a survival analysis were performed using all the available data in the electronic clinical records of patients with a diagnosis of pancreatic cancer in a Hospital of Specialties of QuitoEcuador between 2007 and 2017. The included patients were those coded according to the ICD 10 between C25.0 and C25.9. Our univariate analysis calculated frequencies, measures of central tendency and dispersion. Through the Kaplan-Meier method we estimated the median time of survival and analyzed the difference in survival time among the different categories of our included variables. These differences were shown through the log rank test.

Results: A total of 357 patients diagnosed with pancreatic cancer between 2007 and 2017 were included in the study. More than two-thirds $(69.9 \%)$ of the patients were diagnosed in late stages of the disease. The median survival time for all patients was of 4 months (P25: 2, P75: 8).

\footnotetext{
*Corresponding author: Ruth Jimbo Sotomayor, CISeAL, Quito, Ecuador; and Pontificia Universidad Católica del Ecuador, Quito, Ecuador, Phone: +593995005177, E-mail: rejimbo@puce.edu.ec. https://orcid.org/0000-0001-5016-3834

Andrés Moreno Roca, Hospital Carlos Andrade Marín, Quito, Ecuador; and Facultad de Medicina, Pontificia Universidad Católica del Ecuador, Quito, Ecuador. https://orcid.org/0000-0002-7856-8689 Luciana Armijos Acurio, Centro de Investigación para la Salud en América Latina (CISeAL), Quito, Ecuador; and Facultad de Medicina, Pontificia Universidad Católica del Ecuador, Quito, Ecuador Carlos Céspedes Rivadeneira, Facultad de Medicina, Pontificia Universidad Católica del Ecuador, Quito, Ecuador Carlos Rosero Reyes and Carlos López Ayala, Hospital Carlos Andrade Marín, Quito, Ecuador
}

Conclusions: The statistically significant difference of survival time between types of treatment is the most relevant finding in this study, when comparing to all other types of treatments.

Keywords: Ecuador; pancreatic cancer; survival analysis.

\section{Introduction}

Pancreatic neoplasms are associated with one of the higher mortalities around the world [1, 2]. It is the 14th most prevalent neoplasm and the seventh cause of death for neoplasm worldwide [3]. According to GLOBOCAN 2018, the age-standardized incidence rate (ASR) of pancreatic cancer is 5.5 per 100,000 men, and 4.0 per 100,000 women. ASR mortality is 5.1 per 100,000 men and 3.8 per 100,000 women [4]. In Ecuador, there were 373 deaths due to pancreatic neoplasms in 2017, according to the register of Instituto Nacional de Estadísticas y Censos (INEC) [5].

The risk factors for developing pancreatic neoplasms include genetics, tobacco and alcohol consumption, obesity, and diabetes [6]. The time it takes to develop a pancreatic neoplasm is considered to start between 10 and 17 years before the final stages of the disease. Early identification is associated with a better prognosis $[7,8]$.

The symptoms depend on the localization of the tumor and the stage of the disease. The most common symptoms include abdominal pain, jaundice, nausea, vomit, and steatorrhea [8]. The mean medial survival rate at 5 years is $9 \%$ in all stages of the disease according to the American Society of Cancer between 2008 and 2014 [9].

Today, there is no recommendation for routine screenings of pancreatic neoplasms because of its lower incidence in the general population [3]. The stage of the disease at diagnosis is the most important factor associated with survival rates in patients [1]. Less than $20 \%$ of the patients can receive surgical treatment considering the diagnosis is usually made in the late stages of the disease $[3,9,10]$. Other types of treatments include chemotherapy, radiotherapy, and others [11]. 


\section{Methods}

\section{Study design}

A retrospective cohort study was conducted. The case records of patients with a histologic diagnosis of pancreatic cancer, treated within the time frame of 2007 and 2017, were reviewed. The included patients were those coded according to the ICD 10 between C25.0 and C25.9.

\section{Data sources}

The study uses secondary data from the electronic clinical records of patients with a diagnosis of pancreatic cancer in a Hospital of Specialties in Quito-Ecuador between 2007 and 2017.

\section{Population}

Patients with a diagnosis of pancreatic cancer in a Hospital of Specialties were included and were coded according to the ICD 10 between C25.0 and C25.9.

\section{Statistical analysis}

The sociodemographic and clinical characteristics of the population are described above. Using the Kaplan-Meier survival curves followed by the logrank tests, the survival time after initial diagnosis among the different categories of included variables were calculated (type of treatment, type of cancer, and stage of diagnosis). An analysis was carried out by performing Cox proportional hazard regression models that compared survival time for each variable in order to determine the variables that had a $\mathrm{p}$ value of 0.25 or under. Variables that met the criteria or any other variable that was considered clinically relevant into our saturated Cox regression model were included. By backward variable reduction, a final model was obtained. A sensitivity analysis was furtherly performed.

Stata Version 14 and Microsoft Excel were used for the statistical analysis of this study.

\section{Bioethical considerations}

All the information came from secondary data. In Ecuador, the use of public databases with secondary data does not require previous approval by any ethics committee. The database was properly anonymized before its use.

\section{Results}

For the period of time of the study, 357 clinical records of patients with pancreatic cancer were found. The mean age in pancreatic cancer patients was 62.7 years old (SD 14.3), with the predominance of females $(55.74 \%)$. The most frequent personal history was smoking and alcohol consumption (12 and $51.8 \%$, respectively), and $60 \%$ of patients had a history
Table 1: Sociodemographic and clinical characteristics of the population.

\begin{tabular}{|c|c|}
\hline Variable & $\mathrm{n}, \%$ \\
\hline Mean Age (Range, years) & $62.75(19-92)$ \\
\hline \multicolumn{2}{|l|}{ Gender } \\
\hline Female & 199 (55.74\%) \\
\hline Male & $158(44.26 \%)$ \\
\hline \multicolumn{2}{|l|}{ Education } \\
\hline Primary & 157 (43.98\%) \\
\hline Secondary & $110(30.81 \%)$ \\
\hline Higher education & $90(25.21 \%)$ \\
\hline \multicolumn{2}{|l|}{ Alcohol history } \\
\hline Yes & $129(36.1 \%)$ \\
\hline No & $228(63.9 \%)$ \\
\hline \multicolumn{2}{|l|}{ Smoking history } \\
\hline Yes & $43(12 \%)$ \\
\hline No & $314(88 \%)$ \\
\hline \multicolumn{2}{|l|}{ Diabetes history } \\
\hline Yes & $216(60.5 \%)$ \\
\hline No & $141(39.5 \%)$ \\
\hline \multicolumn{2}{|l|}{ Clinical sign of admission } \\
\hline Abdominal pain & $182(50.98 \%)$ \\
\hline Jaundice & $104(29.13 \%)$ \\
\hline Abdominal mass & 39 (10.92\%) \\
\hline Vomit & $19(5.32 \%)$ \\
\hline Weight loss & $13(3.64 \%)$ \\
\hline \multicolumn{2}{|l|}{ Ca 19-9 } \\
\hline Positive & $254(68.63 \%)$ \\
\hline Negative & $104(29.13 \%)$ \\
\hline No data & $8(2.24 \%)$ \\
\hline \multicolumn{2}{|l|}{ Type of cancer } \\
\hline Endocrino & $39(10.2 \%)$ \\
\hline Exocrino & $318(89.8 \%)$ \\
\hline \multicolumn{2}{|l|}{ Anatomical location } \\
\hline Head & $211(59.10 \%)$ \\
\hline Tail & $35(9.80 \%)$ \\
\hline Body & $70(19.61 \%)$ \\
\hline Others & $41(11.49 \%)$ \\
\hline \multicolumn{2}{|l|}{ Stage of cancer } \\
\hline 0 & $1(0.28 \%)$ \\
\hline I & $63(17.65 \%)$ \\
\hline II & $46(12.89 \%)$ \\
\hline III & $54(15.13 \%)$ \\
\hline IV & $193(54.06 \%)$ \\
\hline \multicolumn{2}{|l|}{ Type of treatment* } \\
\hline Combined & 40 (11.20\%) \\
\hline Palliative & $155(43.42 \%)$ \\
\hline None & 38 (10.64\%) \\
\hline Chemotherapy & 27 (7.56\%) \\
\hline Surgical & 97 (27.17\%) \\
\hline
\end{tabular}

of diabetes. The main sign of admission was abdominal pain (50.98\%). Sixty-eight percent $\%$ of patients had a positive antigen Ca 19-9. Adenocarcinoma was the most frequent type of cancer $(56.86 \%)$, the most frequent location was the head of the pancreas $(59.10 \%)$. More than two-thirds $(69.19 \%)$ of 
the patients were diagnosed in the late stages of the disease, we account for stages III and IV that represented 15.13 and $54.06 \%$, respectively, as of late stages for pancreatic neoplasm. Of these $38.3 \%(n=137)$ patients received surgical treatment, in this group $27.17 \%$ had surgery alone and $11.2 \%$ had surgery plus another treatment (Table 1).

Kaplan-Meier survival curves were created and then followed by a logrank test to compare survival functions. These tests showed a statistically significant difference in survival between groups of treatment $(\mathrm{p}<0.001)$. Survival time from the moment of diagnosis was highest among the group that received surgical treatment at 1, 2, 5 years, and at the end of follow-up (Table 2). The lowest survival proportion for this group was of 0.51 at 3 years of follow up, compared to zero when receiving no treatment or palliative care, 0.16 when receiving chemotherapy, and 0.29 when receiving combined treatment.

The overall survival rate based on treatment modality is shown in Figure 1. The median survival time from the moment of diagnosis for no treatment was 2 months, for chemotherapy 5 months, for surgical treatment 17 months, for combined therapy 7 months, and for palliative care 2 months.

The overall survival rates based on the type of cancer are shown in Figure 2. There is no statistically significant difference in survival rates if the patient was diagnosed with exocrine cancer compared to endocrine cancer $(\mathrm{p}=0.605)$.

The stage at which the cancer was detected clearly marks a difference in survival rate. The median survival time was 14 months for stage II, 5 months for stage III, and 2 months for stage IV.

An unadjusted Cox Regression model that analyzed the relationship between survival time and age, gender, stage at diagnosis, type of cancer, and type of treatment was done to determine the variables that had a $p$ value of 0.25 or under. A saturated model which included stage of diagnosis and type of treatment was performed with variables that met the inclusion criteria. We also included the type of cancer due to its clinical relevance. By backward

Table 2: Survival time according to type of treatment.

\begin{tabular}{|c|c|c|c|c|c|c|}
\hline Interval, months & Beg. Total & Deaths & Lost & Survival & Standard error & [95\% Conf. Int.] \\
\hline \multicolumn{7}{|l|}{ No treatment } \\
\hline $0-12$ & 38 & 38 & 0 & 0.0000 & - & \\
\hline \multicolumn{7}{|l|}{ Chemotherapy } \\
\hline $0-12$ & 27 & 20 & 1 & 0.2453 & 0.0836 & 0.10430 .4174 \\
\hline $12-24$ & 6 & 2 & 0 & 0.1635 & 0.0730 & 0.05310 .3274 \\
\hline $24-36$ & 4 & 0 & 1 & 0.1635 & 0.0730 & 0.05310 .3274 \\
\hline $36-48$ & 3 & 0 & 2 & 0.1635 & 0.0730 & 0.05310 .3274 \\
\hline $60-72$ & 1 & 0 & 1 & 0.1635 & 0.0730 & 0.05310 .3274 \\
\hline \multicolumn{7}{|l|}{ Surgical treatment } \\
\hline $0-12$ & 97 & 23 & 29 & 0.7212 & 0.0494 & 0.61100 .8051 \\
\hline $12-24$ & 45 & 10 & 11 & 0.5386 & 0.0620 & 0.41020 .6508 \\
\hline $24-36$ & 24 & 1 & 4 & 0.5141 & 0.0639 & 0.38320 .6304 \\
\hline $36-48$ & 19 & 0 & 5 & 0.5141 & 0.0639 & 0.38320 .6304 \\
\hline $48-60$ & 14 & 0 & 5 & 0.5141 & 0.0639 & 0.38320 .6304 \\
\hline $72-84$ & 9 & 0 & 5 & 0.5141 & 0.0639 & 0.38320 .6304 \\
\hline $84-96$ & 4 & 0 & 1 & 0.5141 & 0.0639 & 0.38320 .6304 \\
\hline $108-120$ & 3 & 0 & 1 & 0.5141 & 0.0639 & 0.38320 .6304 \\
\hline $132-144$ & 2 & 0 & 1 & 0.5141 & 0.0639 & 0.38320 .6304 \\
\hline $144-156$ & 1 & 0 & 1 & 0.5141 & 0.0639 & 0.38320 .6304 \\
\hline \multicolumn{7}{|c|}{ Combined treatment } \\
\hline $0-12$ & 40 & 24 & 5 & 0.3600 & 0.0784 & 0.21200 .5103 \\
\hline $12-24$ & 11 & 2 & 1 & 0.2914 & 0.0770 & 0.15310 .4448 \\
\hline $24-36$ & 8 & 0 & 1 & 0.2914 & 0.0770 & 0.15310 .4448 \\
\hline $36-48$ & 7 & 1 & 2 & 0.2429 & 0.0780 & 0.10990 .4037 \\
\hline $48-60$ & 4 & 0 & 1 & 0.2429 & 0.0780 & 0.10990 .4037 \\
\hline $60-72$ & 3 & 0 & 3 & 0.2429 & 0.0780 & 0.10990 .4037 \\
\hline \multicolumn{7}{|l|}{ Palliative care } \\
\hline $0-12$ & 155 & 151 & 3 & 0.0163 & 0.0102 & 0.00390 .0471 \\
\hline 1224 & 1 & 1 & 0 & 0.0000 & - & \\
\hline
\end{tabular}




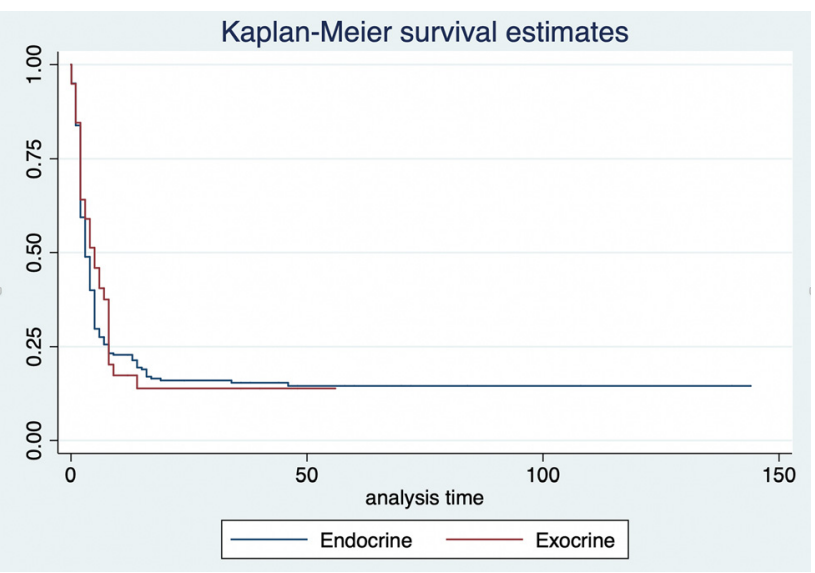

Figure 1: Kaplan-Meier estimates by type of cancer.

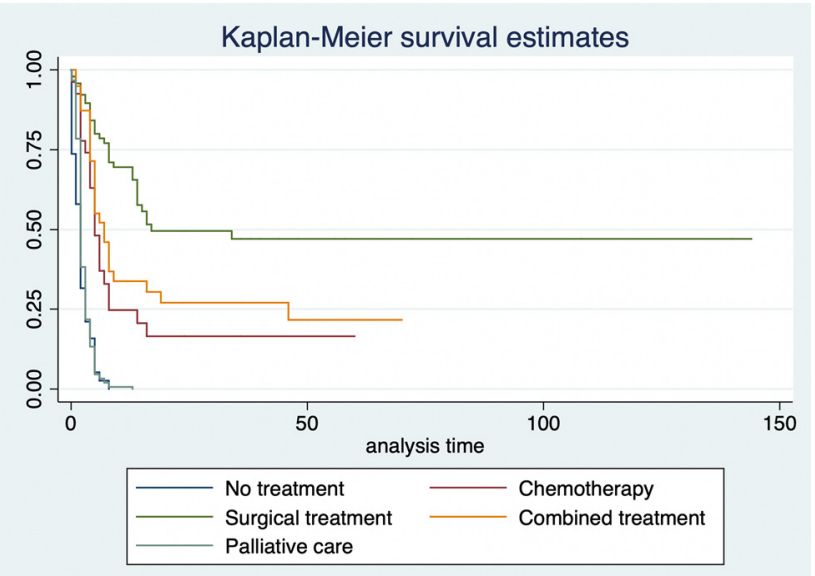

Figure 2: Kaplan-Meier estimates by type of treatment.

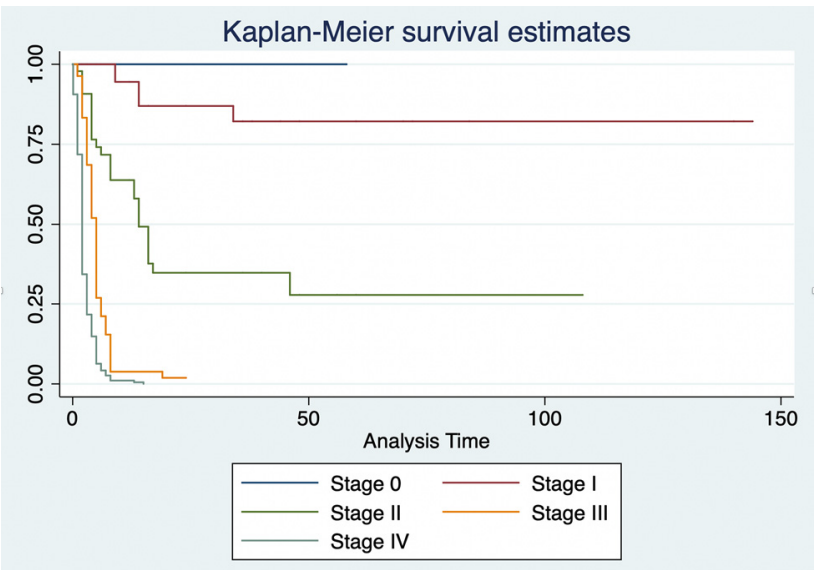

Figure 3: Kaplan-Meier estimates by stage of diagnosis.

variable reduction, a final model which included stage of diagnosis and type of treatment was chosen. The researchers furtherly performed a sensitivity analysis by
Table 3: Final Cox regression model.

\begin{tabular}{lrrrr}
\hline Stage at diagnosis & Hazard ratio & p-Value & $\begin{array}{r}\text { 95\% Confidence } \\
\text { interval }\end{array}$ \\
\hline Stage 1 & 1 & & & \\
Stage 2 & 7.288759 & 0.000 & 2.77248 & 19.16191 \\
Stage 3 & 25.36602 & 0.000 & 9.354024 & 68.78695 \\
Stage 4 & 44.48086 & 0.000 & 16.30272 & 121.363 \\
Type of treatment & & & & \\
No treatment & 1 & & & \\
Chemotherapy & 0.5537656 & 0.036 & 0.31873 & 0.9621194 \\
Surgery & 0.4945155 & 0.013 & 0.2837633 & 0.8617942 \\
Combined & 0.3400392 & 0.000 & 0.2015927 & 0.5735658 \\
Palliative care & 0.780844 & 0.177 & 0.5454472 & 1.11783 \\
\hline
\end{tabular}

eliminating all patients diagnosed at stage 0 , which significantly changed our results, leading us to exclude stage 0 patients. It was found that when adjusting for stage of cancer, there is a 0.49 Hazard Ratio when patients are treated with surgery compared to no treatment $(\mathrm{p}=0.013)$ and of 0.34 when patients are treated with both surgery and chemotherapy $(\mathrm{p}<0.001)$ as described in Table 3.

\section{Discussion}

New diagnosis techniques and treatments have been discovered for pancreatic neoplasms in the last decade. Despite all these advantages, the survival rates have not improved [3, 12].

In our study, $69.9 \%$ of the patients were diagnosed in the late stages of the disease. This could be compared with McGuigan's review of patients, who noted that this was a result of the unspecific symptoms and the rapid invasion of blood vessels [3].

The mean survival rate of patients at 5 years was 2-9\% in a study performed in Shanghai [12], comparable to a 14\% survival rate in our study [13].

The study results show a survival time of 14 months from the moment of diagnosis for patients at stage III compared with only two months in patients at stage IV [14], this data could be compared to the results of Cancer Research UK in which the mean survival time for patients at stage III was 6-11 months and 2-6 months for patients at stage IV [13]. Other studies have found similar results [15-17].

Surgery was associated with the best survival time in patients with pancreatic neoplasms. The mean survival time for patients who underwent surgery was 17 months compared with 5 months for those patients who received chemotherapy. Similar results have been described by Nakeeb [18], Kardosh [16], Nakagawa [19], Brooks [20], and Cancer Research UK [13]. 
A limitation of the study was the exclusion of some patients because their clinical records were incomplete. There were fewer registered patients with pancreatic neoplasms in the years previous to 2010 and some of the patients did not continue with follow-up treatment due to a lack of subsequent appointments at the hospital.

\section{Conclusion}

The statistically significant difference of survival time from the moment of diagnosis between types of treatment is the most relevant finding in this study. When compared to all other types of treatments, surgery yields the best results. An early diagnosis increases the possibility of receiving surgery and having a better survival time in patients.

Research funding: This research received no specific grant from any funding agency in the public, commercial, or notfor-profit sectors.

Author contributions: All authors equally contributed to this manuscript, have accepted responsibility for the entire content, and approved its submission.

Competing interests: Andrés Moreno Roca, Luciana Armijos Acurio, Ruth Jimbo Sotomayor, Carlos Céspedes Rivadeneira, Carlos Rosero Reyes, Carlos López Ayala, declares no conflict of interest.

Ethical approval: All procedures performed in studies involving human participants were in accordance with the ethical standards of the institutional and/or national research committee (Hospital Carlos Andrade Marín) and with the 1964 Helsinki declaration and its later amendments or comparable ethical standards. The study was approved by hospital authorities and the Human Research Ethics Committee.

\section{References}

1. Carrato A, Falcone A, Ducreux M, Valle JW, Parnaby A, Djazouli K, et al. A systematic review of the burden of pancreatic cancer in Europe: real-world impact on survival, quality of life and costs. J Gastrointest Cancer 2015;46:201-11.

2. Ryan DP, Hong TS, Bardeesy N. Pancreatic adenocarcinoma. N Engl J Med 2014;371:1039-49.

3. McGuigan A, Kelly P, Turkington RC, Jones C, Coleman HG, McCain RS. Pancreatic cancer: a review of clinical diagnosis, epidemiology, treatment and outcomes. World J Gastroenterol 2018;24:4846-61.

4. Ferlay J, Colombet M, Soerjomataram I, Mathers C, Parkin DM, Piñeros $M$, et al. Estimating the global cancer incidence and mortality in 2018: GLOBOCAN sources and methods. Int J Canc 2019.
5. Instituto Nacional de Estadística y Censos (INEC). Principales causas de Mortalidad en Ecuador. INEC; 2014. https://www. ecuadorencifras.gob.ec/institucional/home/.

6. Lin QJ, Yang F, Jin C, Fu DL. Current status and progress of pancreatic cancer in China. World J Gastroenterol 2015;21:7888-8003.

7. Yachida S, Jones S, Bozic I, Antal T, Leary R, Fu B, et al. Distant metastasis occurs late during the genetic evolution of pancreatic cancer. Nature 2010;467:1114-7. 20981102.

8. Almond LM, Jaunoo S, European Surgery. Diagnosis and management of pancreatic trauma. Eur Surg - Acta Chir Austriaca 2009;41. https://doi.org/10.1007/s10353-009-0485-6.

9. American Cancer Society. Survival rates for pancreatic cancer. ACS; 2016. https://www.cancer.org/cancer/pancreatic-cancer/ detection-diagnosis-staging/survival-rates.html.

10. Michalski CW, Kleeff J, Wente MN, Diener MK, Büchler MW, Friess $\mathrm{H}$. Systematic review and meta-analysis of standard and extended lymphadenectomy in pancreaticoduodenectomy for pancreatic cancer. Br J Surg 2007;94:265-73.

11. Long J, Luo G, Xiao Z, Liu Z, Guo M, Liu L, et al. Cancer statistics: current diagnosis and treatment of pancreatic cancer in Shanghai, China. Cancer Lett 2014;346:273-7.

12. Chu LC, Goggins MG, Fishman EK. Diagnosis and detection of pancreatic cancer. Canc J;23:333-42. https://doi.org/10.1097/ ppo.0000000000000290.

13. Luo J, Xiao L, Wu C, Zheng Y, Zhao N. The incidence and survival rate of population-based pancreatic cancer patients: Shanghai Cancer Registry 2004-2009. PloS One 2013;8:e7652.

14. Cancer Research UK. Survival pancreatic cancer. Cancer Research UK; 2018. https://www.cancerresearchuk.org/healthprofessional/cancer-statistics/statistics-by-cancer-type/ pancreatic-cancer.

15. Wahutu M, Vesely SK, Campbell J, Pate A, Salvatore AL, Janitz AE. Pancreatic cancer: a survival analysis study in Oklahoma. J Okla State Med Assoc 2016;109:391-8. PMID: 27885308; PMCID: PMC5119763.

16. Kardosh A, Lichtensztajn DY, Gubens MA, Kunz PL, Fisher GA, Clarke CA, et al, A California Population-Based Study. Long-term survivors of pancreatic cancer: a California population-based study. Pancreas 2018;47:958-66.

17. Vincent A, Herman J, Schulick R, Hruban RH, Goggins M. Pancreatic cancer. Lancet 2011;378:607-20.

18. El Nakeeb A, El Sorogy M, Ezzat H, Said R, El Dosoky M, Abd El Gawad M, et al. Predictors of long-term survival after pancreaticoduodenectomy for peri-ampullary adenocarcinoma: a retrospective study of 5-year survivors. Hepatobiliary Pancreat Dis Int 2018;17:443-9.

19. Nakagawa K, Akahori T, Nishiwada S, Nagai M, Nakamura K, Tanaka T, et al. Prognostic factors for actual long-term survival in the era of multidisciplinary treatment for pancreatic ductal adenocarcinoma. Langenbeck's Arch Surg 2018;403: 693-700.

20. Brooks JC, Shavelle RM, Vavra-Musser KN. Life expectancy in pancreatic neuroendocrine cancer. Clin Res Hepatol Gastroenterol 2019;43:88-97.

Supplementary Material: The online version of this article offers reviewer assessments as supplementary material (https://doi.org/ 10.1515/iss-2020-0030). 